\title{
Pression Foncière En Milieu Rural Ivoirien : Quelles Stratégies Adoptent Les Paysans Baoulé Réinstallés Dans La Forêt Des “Tos"' À Bouaflé ?
}

\author{
Konan Affouet Geneviève Épse Téni, Doctorante \\ Institut de Géographie Tropicale, \\ Université Félix Houphouët-Boigny, Abidjan, Côte d'Ivoire \\ Aloko-N'guessan Jérôme, Directeur de recherches (Cames) \\ Université Félix Houphouët-Boigny, Abidjan, Côte d'Ivoire
}

Doi:10.19044/esj.2019.v15n35p167 URL:http://dx.doi.org/10.19044/esj.2019.v15n35p167

\section{Résumé}

La construction du barrage hydro-électrique de Kossou en 1970 a nécessité la réinstallation de 11000 paysans baoulé, en majorité Ayaou, dans la forêt des "Tos", située dans le département de Bouaflé. Cette zone enregistrait au préalable une forte concentration de populations agricoles qui exerçaient une pression sur l'espace cultivable. Avec l'arrivée massive des déguerpis, cette pression foncière s'est accentuée au fil des années, au point où l'espace cultivable s'est raréfié. Cette étude se propose d'analyser les stratégies mises en place par les déplacés face aux difficultés d'accès à l'espace cultivable. Pour ce faire, la démarche méthodologique repose sur la recherche documentaire et l'enquête de terrain (observation directe et questionnaire) réalisée en Mai 2015. La technique du choix raisonné fut retenue pour le choix des villages enquêtés et la méthode des quotas pour celui des chefs de ménage. Sur cette base, 1/10è soit 504 chefs de ménage ont été enquêtés. Il ressort de cette étude que pour faire face à cette contrainte foncière, certains individus $(50,79 \%)$ ont migré vers d'autres régions du pays à la recherche de mieux être. Pour ceux qui sont restés sur le site d'accueil, l'heure est aujourd'hui à une réorientation géographique des objectifs de production. Elle consiste pour certains, à repenser le modèle cultural par l'intensification (54\%) et l'association culturale (95\%) sur les espaces en leur possession. D'autres exploitants (41\%), se consacrent aux activités alternatives à l'agriculture génératrices de revenus telles que l'élevage et le secteur informel.

Mots clés : Stratégie Foncière, Développement Agricole, Pression Foncière, Contrainte Foncière, Forêt Des “Tos", Côte d'Ivoire 


\title{
Land Pressure in Rural Ivory Coast: What Strategies Adopt the Baoulé Farmers Resettled in the Forest of "Tos"' in Bouaflé?
}

\author{
Konan Affouet Geneviève Épse Téni, Doctorante \\ Institut de Géographie Tropicale, \\ Université Félix Houphouët-Boigny, Abidjan, Côte d'Ivoire \\ Aloko-N'guessan Jérôme, Directeur de recherches (Cames) \\ Université Félix Houphouët-Boigny, Abidjan, Côte d'Ivoire
}

\begin{abstract}
The construction of the Kossou hydroelectric dam in 1970 necessitated the resettlement of 11,000 Baoule farmers, most of them Ayaou, in the forest of "Tos", located in the department of Bouaflé. This zone previously recorded a high concentration of agricultural populations which exerted pressure on the arable land. With the massive arrival of the evaders, this land pressure has increased over the years, to the point where the cultivable space has become rarefied. This study proposes to analyze the strategies put in place by the displaced people with regard to the difficulties of access to the cultivable space. To do this, the methodological approach is based on the documentary research and the field survey (direct observation and questionnaire) conducted in May 2015. The reasoned choice technique was chosen for the choice of villages surveyed and the quota method for the heads of households. On this basis, 1 / 10th or 504 household heads were surveyed. According to this study, to cope with this land constraint, some individuals $(50.79 \%)$ migrated to other regions of the country in search of better life. For those who remained on the site of reception, the time is today to a geographical reorientation of the objectives of production. For some, it involves rethinking the cultural model through intensification $(54 \%)$ and cultural association $(95 \%)$ on the areas in their possession. Other farmers (41\%) are engaged in alternative farming, income-generating activities such as livestock and the informal sector.
\end{abstract}

Keywords: Land Strategy, Agricultural Development, Land Pressure, Land Constraint, Forest Of "Tos", Ivory Coast 


\section{Introduction}

La forêt des “'Tos”' à Bouaflé est une zone retenue pour accueillir les populations sinistrées du fait de la construction du barrage hydroélectrique de Kossou en 1970. Cette forêt a été classée depuis 1937 en une aire protégée et représentait 16200 hectares (De La Vaissière, 1978 : 76). Des études menées par De La Vaissière (op cité : 76-78) et Dibi et al (2008:18), révèlent que ce site choisi pour installer ces villages était une zone déjà densément occupée. Ori B (1984a : 6-7), confirmera cette assertion en révélant que la forêt des "Tos" a connu son premier déclassement partiel en 1951 au profit des villages Gouro Blanfla et Konéfla situés au Sud-est. Certaines notabilités de ces dits villages ont créé de grandes plantations dans une partie de la forêt des “Tos"'. Au Nord-est, des Mossis ${ }^{12}$ installés depuis de longues dates infiltrent ladite forêt. De plus, 370 hectares de cette forêt ont été mis à la disposition de la SODEFOR ${ }^{13}$ pour la création des plantations de teck (De La Vaissière, op cité : 76).

Dans ces conditions de saturation foncière, l'État a encore procédé à un deuxième déclassement par un décret en date du 28 février 1970. Celui-ci porte sur la propriété de 20000 hectares. Elle a été confiée à l'Autorité pour l'Aménagement de la Vallée du Bandama (AVB), chargée de réinstaller les populations déguerpies estimées à 11000 habitants en 1970 (Ori B, 1984a : 78). Alors que ces secteurs ne pouvaient accueillir que des agglomérations d'environ 1200 personnes ; normes retenues en raison de la pression foncière déjà existante (Lassailly-Jacob, 1989 : 141). L'AVB a disposé d'un terrain agricole d'une superficie de 11700 hectares sur lequel les populations déplacées devraient installer leurs plantations (De La Vaissière ,1978 : 78). Aujourd'hui, elles continuent de pratiquer l'agriculture sur cet espace restreint. Or, plus de quarante années après leur installation, le site d'accueil des déplacés connaît une pression démographique (INS, 2014). Comme toute attente, l'accès à un nouvel espace cultivable est devenu incertain. La population est donc en quête perpétuelle de solution pour se maintenir dans le nouvel espace d'accueil. Face à cette situation, nous nous posons la question qui suit :

Quelles sont les stratégies agricoles développées par ces populations réinstallées face aux contraintes foncières ?

L'objectif principal de cet article est d'analyser les stratégies mises en place par la population dans la forêt des "Tos", face à la pression foncière. De façon spécifique, quels sont les facteurs de la pression foncière et quelles sont les destinations privilégiées par les populations qui tentent d'échapper à cette pression? Quelles sont les techniques culturales recourues par les exploitants ? Et enfin, quelles sont les reconversions qui se sont opérées ?

\footnotetext{
${ }^{12}$ Mossi : populations d'origine Burkinabé (ex Haute-Volta)

${ }^{13}$ SODEFOR : Société de Développement des Forêts
} 
La méthodologie suivante a permis d'apporter des éléments de réponse à ces préoccupations.

\section{Matériels Et Méthodes \\ $1 \quad$ L'espace D'étude}

L'étude a porté sur les 21 villages réinstallés dans le département de Bouaflé à la faveur de l'Aménagement de la Vallée du Bandama (AVB). Ces villages ont été regroupés en huit villages modernes (Nangrékro, N'dènoukro, Attossé, N'douffoukankro, Blè, Akoviébo, Diacohou-sud et Bénou) pour une population estimée à 11000 habitants en 1970 (Ori B, 1984: 7-8) (carte 1).

Carte 1 : Présentation de l'espace d'étude

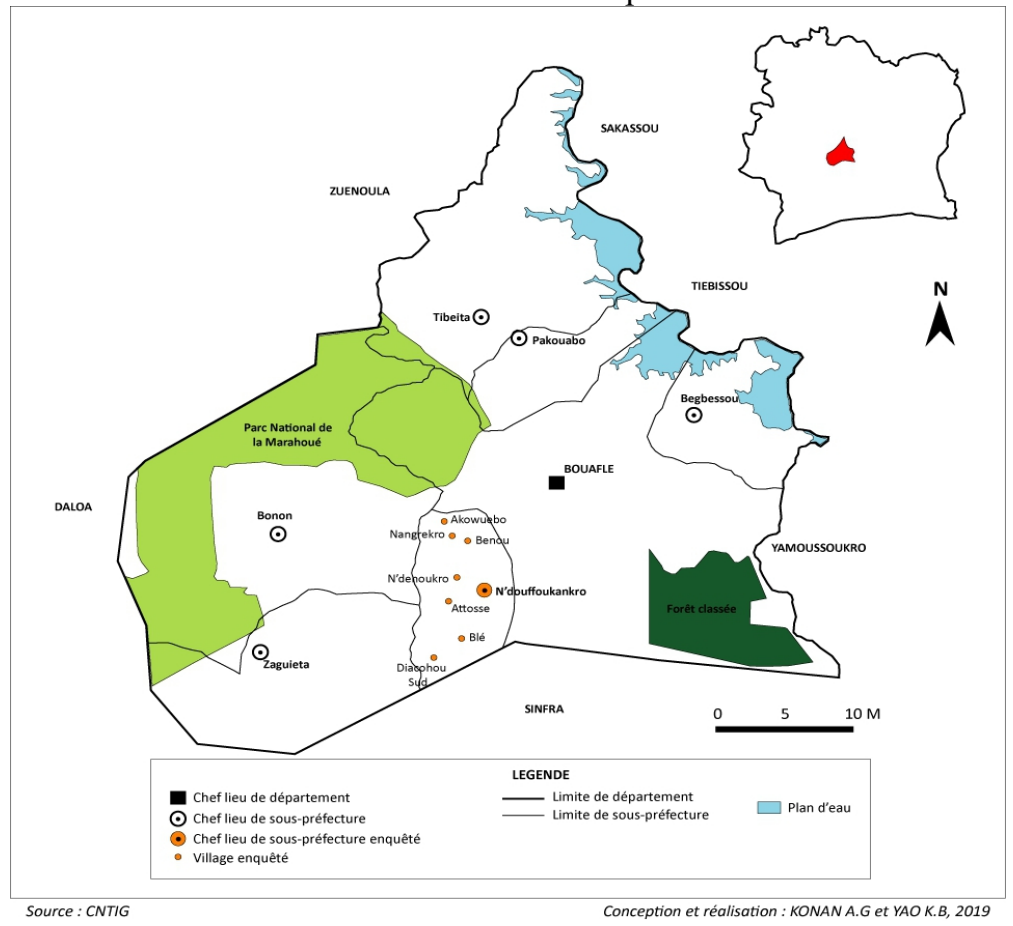

Ils sont situés dans la forêt appartenant aux Gouro de Toho, dont la déformation a donné "Tos". Cet espace est situé au Sud-ouest du département de Bouaflé. Il est confiné entre les axes Bouaflé-Daloa, passant par Bonon et Bouaflé-Sinfra. Les populations sont constituées en majorité par les Ayaou, un sous-groupe ethnique des Baoulé. À l'exception d'un seul (Bénou) qui est peuplé de Yaourè. Ils sont situés sur un relief de bas plateaux (200 ou 300 m) et de hauts plateaux $(400 \mathrm{~m})$ au sol ferralitique fortement désaturé sous pluviométrie moyenne (VENNETIER et al in atlas de Côte d'Ivoire, 1978). Le climat tropical de type "baouléen" est caractérisé par une longue période de sécheresse. La végétation est constituée d'une mosaïque de savanes, de forêts claires et de forêts denses semi-décidues. La zone est arrosée par le 
Bandama rouge (Marahoué) et de nombreux cours d'eau (Baha, Ouréné, Bôlè, Zabré, Blo N'Zué, etc.).

\section{Les méthodes de collecte des données}

Les méthodes de collecte des données reposent sur la recherche documentaire et l'enquête de terrain. La documentation a porté essentiellement sur la pression foncière et les stratégies développées par les populations face à ce phénomène ainsi que sur la dynamique sociodémographique dans les villages enquêtés. L'article de De La Vaissière (1978) a été d'un grand intérêt compte tenu des questions de stratégies pionnières et d'intensification dans la forêt des "Tos" qu'il aborde ; de même que celui de Ori (1984) sur les données socio-démographiques dans les villages de l'Ayaou Sud. L'enquête de terrain s'est effectuée en mai 2015, dans les huit villages réinstallés. Elle a permis de faire l'observation directe, des entretiens et l'enquête par un questionnaire. L'observation qui est une technique de contact avec le terrain a permis de se familiariser avec le site d'étude en sillonnant les différents villages enquêtés. Elle a permis d'apprécier les combinaisons de culture et l'ampleur de la pression humaine sur le foncier rural, de répertorier les différentes activités économiques et de faire des prises de vue. Quant aux entretiens, les cibles d'enquête retenues sont essentiellement les personnes ressources locales (les chefs de villages/les notables, les présidents des jeunes, les présidentes des femmes, les chefs de ménages). Il était question de s'imprégner des stratégies adoptées par ces paysans face à la pression foncière. Concernant le questionnaire, les investigations ont porté sur tous les huit villages déplacés, anciennement constitués de 21 villages déguerpis. Les questions ont porté sur le profil du chef de ménage (le sexe, l'âge, l'ethnie, la nationalité et la situation professionnelle), l'exploitation (la superficie et la nature des cultures pratiquées), les techniques et méthodes culturales et la stratégie d'occupation du sol. À cet effet, une fiche d'enquête a été élaborée pour recueillir les informations. Les villages enquêtés comptent 5040 ménages (INS, 2014). Dans l'impossibilité d'enquêter tous ces ménages, un échantillonnage a été recouru. Sur la base de la méthode du choix raisonné, les huit villages réinstallés dans la forêt des "Tos" ont été la cible de l'enquête. Selon la méthode des quotas, il a été trouvé raisonnable d'interroger $1 / 10^{\mathrm{è}}$ des chefs ménages de l'espace d'étude soit 504 chefs de ménages. Ainsi, les critères d'âge, de sexe et de possession d'une exploitation agricole ont été privilégiés.

\section{$3 \quad$ Le traitement des données}

Le logiciel Sphinx a permis de saisir les données d'enquête, de dépouiller le questionnaire et de générer le croisement des informations à travers des tableaux. Les données qualitatives ont été traitées avec le logiciel 
Word. Le logiciel Excel a servi à élaborer les différents tableaux et figures contenus dans cet article. La carte de présentation de l'espace d'étude a été réalisée avec le logiciel Adobe Illustrator CS.

\section{Résultats}

1 Les indicateurs de la pression foncière

La croissance de la population, le retour des déscolarisés à la terre, les crises politiques et les faits de société sont les principaux facteurs explicatifs de la pression foncière.

\subsection{La croissance de la population}

Les populations déplacées représentent une vague migratoire importante pour l'espace “'Tos" déjà densément occupé. C'est une population fixée définitivement et qui a connu une évolution constante dans le temps. Cette croissance a été enregistrée au sein de chaque village enquêté et est confirmée par les résultats des différents recensements de la population (figure $1)$.

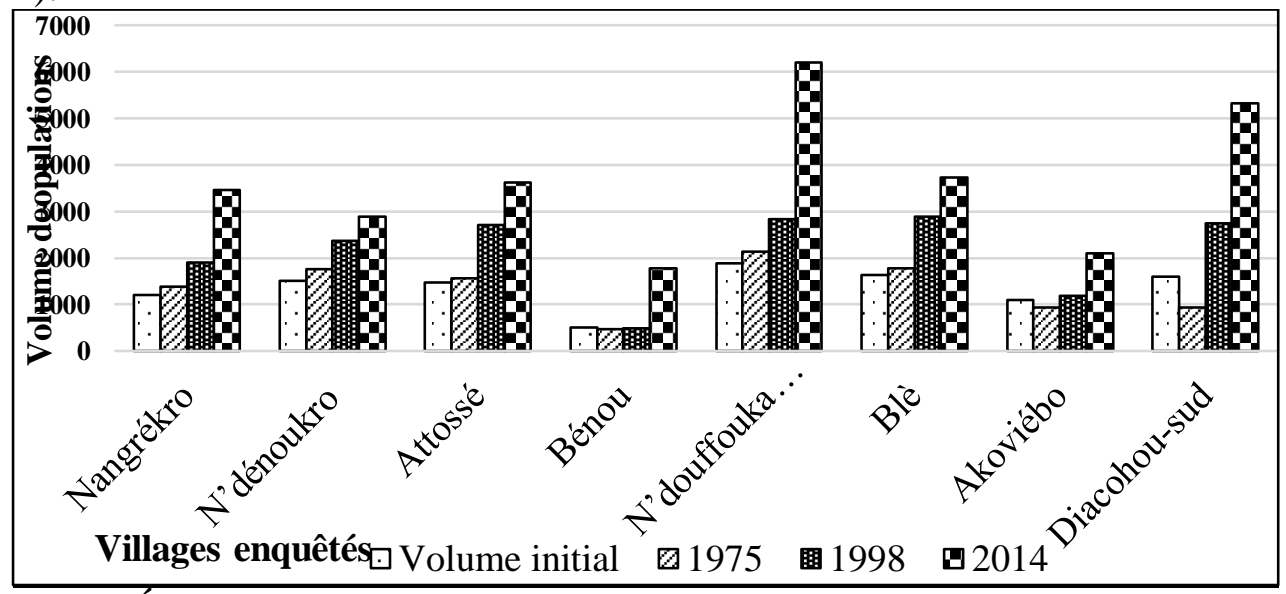

Figure 1 : Évolution de la population par village sur les périodes 1975, 1998 et 2014 (INS, 19751998-2014).

L'analyse de la figure 1 montre que la population initiale dans tous les villages a connu une croissance sur la période 1975-2014. Elle est passée de 10958 habitants en 1975 à 17139 habitants en 1998 pour atteindre 29097 habitants en 2014 ; soit une augmentation de plus du double du volume initial en 2014. Ce croit rapide de la population est une source de pression sur les espaces cultivables. À cela s'ajoutent la forte proportion d'individus dont le retour au village est lié à la déscolarisation, à des faits politiques et de société ; et qui empiètent sur les espaces cultivables disponibles. 


\subsection{Le retour des déscolarisés à la terre}

Le retour des élèves au village après l'échec scolaire accentue la pression sur les terres disponibles. La proportion par village est représentée dans le tableau 1 suivant.

Tableau 1 : La proportion des exploitants déscolarisés par village

Source : Données collectées, 2015

\begin{tabular}{|c|c|c|}
\hline $\begin{array}{c}\text { Déscolarisés } \\
\text { Nom du village }\end{array}$ & Effectifs & Pourcentage (\%) \\
\hline Nangrékro & 25 & 4,96 \\
\hline N'dènoukro & 27 & 5,36 \\
\hline Attossé & 34 & 6,75 \\
\hline Bénou & 45 & 8,93 \\
\hline N'douffoukankro & 22 & 4,36 \\
\hline Blè & 32 & 6,35 \\
\hline Akoviébo & 24 & 4,76 \\
\hline Diacohou-sud & 29 & 5,75 \\
\hline Total déscolarisés & $\mathbf{2 3 8}$ & $\mathbf{4 7 , 2 2}$ \\
\hline Non réponse & 266 & 52,78 \\
\hline Total général & $\mathbf{5 0 4}$ & $\mathbf{1 0 0}$ \\
\hline
\end{tabular}

Les données du tableau 1 montrent qu'un effectif total de 238 exploitants soit $47,22 \%$ des enquêtés sont retournés à la terre après l'échec scolaire. Ce phénomène s'observe dans tous les villages et la population concernée représente $2 / 3$ environ de l'effectif des exploitants enquêtés dans chaque village.

\subsection{Les crises politiques et les faits de société}

Les informations relatives au motif de la présence des enquêtés au village ont permis d'apprécier ces phénomènes (faits politiques et de société) (tableau 2).

Tableau 2 : Motif d'installation des enquêtés au village

\begin{tabular}{|c|c|c|}
\hline Raison installation & Effectif & Pourcentage (\%) \\
\hline Non Scolarisés & 146 & 28,97 \\
\hline Déscolarisés & 238 & 47,22 \\
\hline Mariage & 27 & 5,35 \\
\hline Héritage & 90 & 17,86 \\
\hline Crise socio-politique 2002 & 1 & 0,2 \\
\hline Crise post-électorale 2011 & 1 & 0,2 \\
\hline Retraite & 1 & 0,2 \\
\hline Total & $\mathbf{5 0 4}$ & $\mathbf{1 0 0}$ \\
\hline
\end{tabular}

Source : Données collectées, 2015

Les résultats présentés dans le tableau 2 indiquent que $0,4 \%$ des exploitants sont retournés au village sous la contrainte des crises politiques contre $0,2 \%$ dont le retour est lié à la retraite. 
Enfin, l'ensemble des personnes dont le retour à la terre est dû aux faits de société, à l'échec scolaire et aux crises politiques représente $48 \%$ des enquêtés.

\section{Une mobilité de population liée à la contrainte foncière}

L'émigration enregistrée au sein des villages est l'apanage des exploitants en quête d'espace cultivable pour certains. L'opinion oui ou non des enquêtés sur la question de savoir s'ils ont enregistré en leur sein des membres de la famille partis en aventure, a révélé les résultats suivants (figure 2).

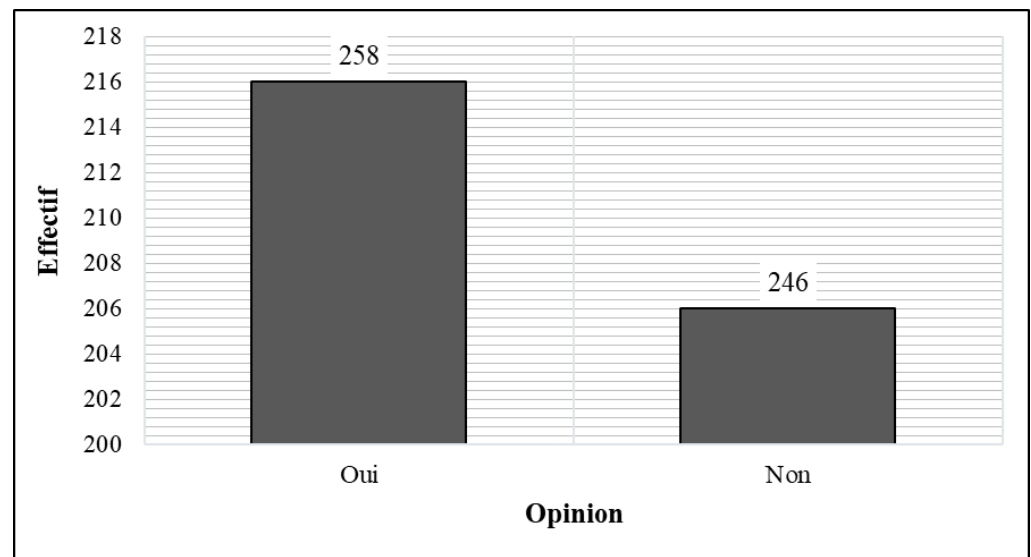

Figure 2 : Opinion des enquêtés sur le départ de parents du village

Les données de la figure 2 ci-dessus montrent que 51\% des ménages enquêtés ont enregistré en leur sein des parents partis du village contre $49 \%$ qui estiment que les membres de la famille sont restés sur place. Le constat qui se dégage est que 01 ménage sur 02 a eu au moins un membre de sa famille parti en aventure à la recherche de bien-être. Ces personnes qui sont parties du village s'orientent soit vers des zones rurales ou urbaines (tableau 3 ).

Tableau 3 : L'effectif des migrants en fonction des différents lieux de destination

\begin{tabular}{|c|c|c|}
\hline \multicolumn{2}{|c|}{ Destination } & \multicolumn{2}{c|}{ Nombre d'exploitants } \\
\hline \multicolumn{2}{|c|}{ Ouest } & 53 \\
\hline Centre-ouest & PNM & 59 \\
\cline { 2 - 3 } & Autres & 32 \\
\hline \multicolumn{2}{|c|}{ Sud-ouest } & 8 \\
\hline Nord-ouest & 8 \\
\hline Sud & 7 \\
\hline Centre & 5 \\
\hline Est & 133 \\
\hline Ancien site & 33 \\
\hline Bouaflé-ville & 33 \\
\hline Abidjan & $\mathbf{3 8 8}$ \\
\hline TOTAL & \\
\hline
\end{tabular}

Source : Données collectées, 2015

PNM : Parc National de la Marahoué 
Les informations dans le tableau 3 ci-dessus indiquent qu'un total de 388 personnes sont parties des villages. Dans leur rang, 321 personnes se sont dirigées vers les zones rurales. Au sein de cette catégorie, 53 personnes ont infiltré le PNM et 133 personnes (représentant près de la moitié des exploitants) sont retournées sur les anciens sites. 66 personnes quant à elles, se sont dirigées vers les centres urbains. Pendant ce temps, les exploitants qui sont restés sur place ont repensé leur modèle cultural.

\section{$3 \quad$ La stratégie foncière adoptée par les exploitants}

La mutation qui a lieu dans le système cultural face à la pression foncière consiste à l'association des cultures, à l'utilisation des intrants et à l'introduction de variétés plus productives

\subsection{L'association des cultures : un moyen de mise à profit de l'espace cultivable}

Les exploitants recourent à l'association des cultures dans cette nouvelle configuration spatiale.

Tableau 4 : Système de culture pratiqué par les exploitants enquêtés

\begin{tabular}{|c|c|c|}
\hline Système de culture pratiqué & Effectifs & Pourcentage (\%) \\
\hline Association de culture & 480 & 95 \\
\hline Monoculture & 24 & 5 \\
\hline Total & 504 & 100 \\
\hline
\end{tabular}

Source : Données collectées, 2015

Les données du tableau montrent que la quasi-totalité des enquêtés 95 $\%$ associent les cultures sous les différentes exploitations contre seulement 5 $\%$ qui pratiquent la monoculture. D'autres recourent à l'utilisation des intrants pour rentabiliser l'espace cultivable disponible.

\subsection{L'agriculture intensive comme stratégie de gestion efficace et rationnelle de l'espace}

Ce système fait intervenir l'utilisation d'intrants. Les opinions oui ou non des exploitants sur la question ont permis d'obtenir les résultats mentionnés dans le tableau 5.

Tableau 5 : Opinion des enquêtés sur l'utilisation des intrants

\begin{tabular}{|c|c|c|}
\hline Opinion & Effectifs & Pourcentage (\%) \\
\hline Oui & 271 & 54 \\
\hline Non & 233 & 46 \\
\hline TOTAL & $\mathbf{5 0 4}$ & $\mathbf{1 0 0}$ \\
\hline
\end{tabular}

Source : Données collectées 2015

L'examen du tableau 5 montre que plus de la moitié des enquêtés (54 $\%$ ) utilisent les intrants sur leurs différentes exploitations. Par contre, $46 \%$ des enquêtés n'en font pas usage au moment où l'utilisation des intrants est 
devenue indispensable pour une bonne production. À cet effet, plusieurs types d'intrants sont utilisés par les exploitants (figure 3). La figure 3 montre qu'au nombre des intrants utilisés, l'emploi des herbicides est dominant avec $27 \%$ des exploitants qui s'adonnent à son utilisation. Il est suivi des insecticides qui sont utilisés par $14 \%$ des exploitants ainsi que les fertilisants (minérale ou organique), utilisés par $13 \%$ des exploitants. Les fongicides sont les moins utilisés. C'est seulement 01 individu (0,2\%) qui en fait usage.

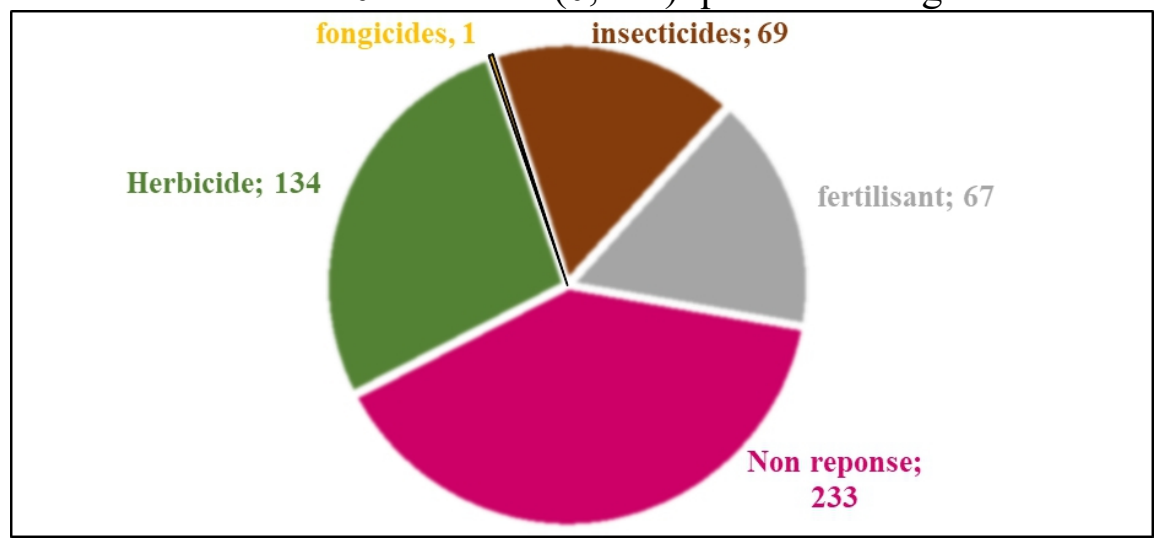

Figure 3 : Répartition des enquêtés selon le type d'intrants utilisé

\subsection{La vulgarisation de nouvelles variétés plus productives dans le système cultural}

Pour donner un nouvel essor au développement de l'agriculture, de nouvelles spéculations plus adaptées à la contrainte foncière sont introduites dans les systèmes culturaux. Il s'agit du maïs hybride dont une ferme pilote a été observée à Blè (photo 1) et du cacao hybride.

Photo 1 : Vue de profil d'une exploitation pilote de maïs hybride à Blè

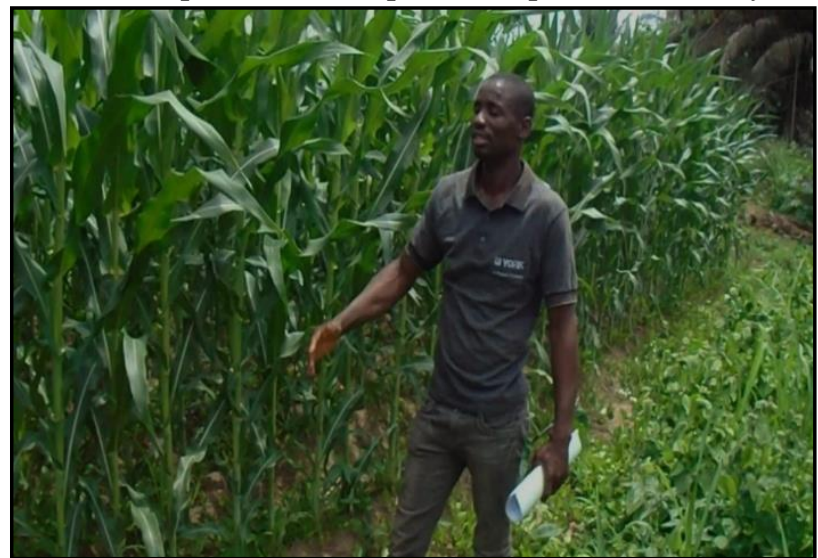

Sur la photo $n^{\circ} 1$, le guide explique les procédés de production de ce type de maïs. Ce champ rentre dans le cadre de la phase expérimentale de la variété hybride et de sa vulgarisation dans les “Tos", espace soumis à une très forte pression foncière. Elle vient répondre à la pression foncière de par son rendement très élevé à l'hectare.

Source : Konan G., Mai, 2015 
La culture du maïs hybride est à sa phase pilote dans les "Tos" au moment de l'enquête. Cette exploitation pilote a été mise en place il y a environ 3 semaines. Elle s'étend sur une superficie de $40 \mathrm{~m}^{2}$. La présentation linéaire de ce champ ainsi que l'espacement des différents plants montrent les exigences particulières qu'impose la production du maïs hybride. Toutefois, ces actions préalables sont appuyées par le développement d'autres activités génératrices de revenus.

\section{Le développement d'activités non agricoles comme alternative à la pression foncière}

Il s'agit des activités non agricoles telles que l'élevage, la pêche et les activités informelles.

\subsection{L'élevage, un secteur le plus convoité}

L'élevage des volailles, des bovins, des porcs, des ovins et des caprins, l'apiculture, la pisciculture et l'élevage des cobayes sont les types rencontrés.

\subsubsection{L'élevage de volailles}

Les exploitants qui s'adonnent à l'élevage de volailles sont au nombre de 31 soit $6 \%$ des enquêtés. Les poulets locaux, les poulets de chair, les pintades et les canards sont les espèces qui sont élevées dans ce type d'élevage (tableau 6).

Tableau 6: Nombre de têtes de volailles élevées par les acteurs selon les catégories

\begin{tabular}{|c|c|c|c|c|c|c|}
\hline \multirow{2}{*}{$\begin{array}{l}\text { Type de volailles } \\
\text { Nombre de têtes }\end{array}$} & \multicolumn{2}{|c|}{ Poulet } & \multicolumn{2}{|c|}{ Pintade } & \multicolumn{2}{|c|}{ Canard } \\
\hline & Effectifs & $\%$ & Effectifs & $\%$ & Effectifs & $\%$ \\
\hline Non réponse & 398 & 94,31 & 421 & 99,76 & 421 & 99,76 \\
\hline Moins de 60 & 9 & 2,13 & & & 1 & 0,24 \\
\hline De 60 à 90 & 6 & 1,42 & 1 & 0,24 & & \\
\hline De 90 à 120 & 4 & 0,95 & & & & \\
\hline De 120 à 150 & & & & & & \\
\hline De 150 à 180 & 3 & 0,71 & & & & \\
\hline De 180 à 210 & 1 & 0,24 & & & & \\
\hline 210 et plus & 1 & 0,24 & & & & \\
\hline TOTAL & 422 & 100 & 422 & 100 & 422 & 100 \\
\hline
\end{tabular}

Les données du tableau montrent que l'élevage de poulets est le type dominant avec un important nombre de têtes en élevage. À cet effet, 09 personnes disposent de plus de 100 à plus de 200 têtes, 06 personnes ont plus de 60 têtes en élevage et 09 disposent au moins de 50 têtes. Respectivement, deux personnes se sont intéressées à l'élevage des pintades et de celui des canards en disposant chacune d'au moins 90 têtes et 60 têtes. Ce qui montre l'intérêt que les exploitants accordent à ce type d'élevage. Les bœufs, les moutons, les cabris et les porcs font aussi parties des activités d'élevage pratiquées. 


\subsubsection{L'élevage bovin, ovin, caprin et porcin}

Dans le tableau 7, la part des individus qui se sont intéressés à ces quatre types d'élevage. Les données de ce tableau montrent que 41 individus (soit $8 \%$ des enquêtés) pratiquent ces quatre types d'élevage ; dont 10 pour le bovin, 03 pour le caprin, 21 pour l'ovin et 07 pour le porcin. Les quantités de têtes élevées sont constituées de 40 têtes de bœufs, 25 têtes de cabris, 260 têtes de moutons et 173 têtes de porcs. Ces données montrent l'importance de ces activités. Puisque 23 individus disposent de 10 têtes et plus de bêtes, tout type confondu en élevage (tableau 7).

Tableau 7: Nombre de têtes disposées par les acteurs selon le type d'élevage

\begin{tabular}{|c|c|c|c|c|c|c|c|c|}
\hline \multirow{2}{*}{$\begin{array}{c}\text { Nbre } \\
\text { Te Têtes }\end{array}$} & \multicolumn{2}{|c|}{ Bovins } & \multicolumn{2}{c|}{ Ovins } & \multicolumn{2}{c|}{ Caprins } & \multicolumn{2}{c|}{ Porcine } \\
\cline { 2 - 9 } & Effectifs & $\%$ & Effectifs & $\mathbf{\%}$ & Effectifs & $\mathbf{\%}$ & Effectifs & $\%$ \\
\hline Non réponse & 494 & 98,10 & 483 & 95,73 & 501 & 99,28 & 497 & 98,57 \\
\hline moins de 4 & 4 & 0,71 & 4 & 0,71 & 1 & 0,24 & 1 & 0,24 \\
\hline De 4 à 6 & 5 & 0,95 & 1 & 0,24 & & & & \\
\hline De 6 à 8 & & & & & & & & \\
\hline De 8 à 10 & 1 & 0,24 & & & 1 & 0,24 & 1 & 0,24 \\
\hline De 10 à 12 & & & 1 & 0,24 & & & & \\
\hline De 12 à 14 & & & 1 & 0,24 & & & & \\
\hline De 14 et plus & & & 14 & 2,84 & 1 & 0,24 & 5 & 0,95 \\
\hline Total & $\mathbf{5 0 4}$ & $\mathbf{1 0 0}$ & $\mathbf{5 0 4}$ & $\mathbf{1 0 0}$ & $\mathbf{5 0 4}$ & $\mathbf{1 0 0}$ & $\mathbf{5 0 4}$ & $\mathbf{1 0 0}$ \\
\hline
\end{tabular}

Source : Données collectées, 2015

\subsubsection{Le développement de l'apiculture}

Au sein des enquêtés, 09 personnes dont 04 femmes pratiquent ce type d'élevage. Elles ont été respectivement rencontrées à Nangrékro avec 01 femme et 03 femmes à N'douffoukankro, 01 personne à Blé et 04 personnes à Diacohou-sud. Elles disposent d'un total de 38 caisses. 04 personnes disposent respectivement de 03 et 05 caisses et 05 personnes disposent chacune de 10 caisses. La pisciculture intègre également les habitudes en élevage.

\subsubsection{L'intégration de la pisciculture dans les pratiques d'élevage}

Les activités qui ont lieu dans ce domaine mobilisent 05 personnes. On enregistre un total de 19 barrages construits dans le cadre de cette activité (photo 2). 
Photo 2 : Vue d'un barrage traditionnel à Diacohou-sud

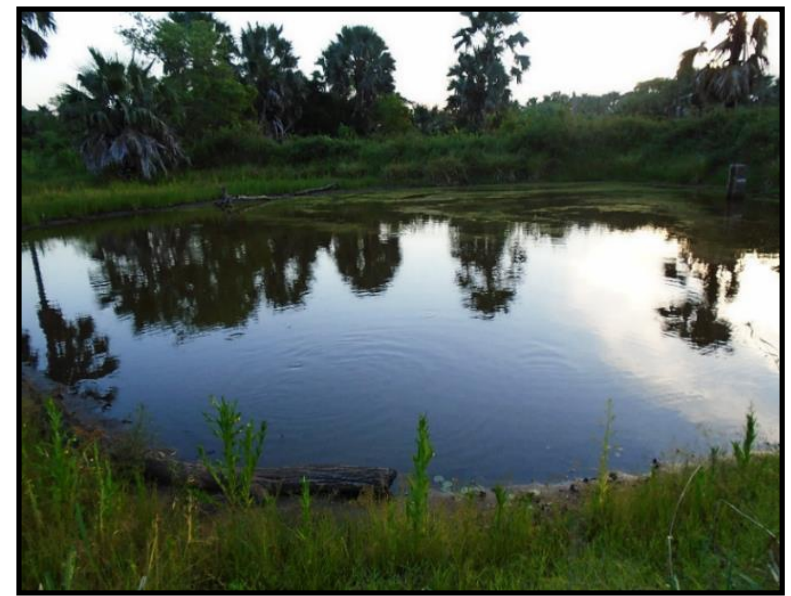

Ce barrage est érigé sur l'un des points d'eau présents dans la région. Il abrite différentes espèces de poissons qui y sont élevées. C'est une activité qui génère des revenus importants aux acteurs.

Source : Konan G., Mai, 2015

Le nombre d'espèces en élevage est estimé à 2600 dans l'ensemble. Le tilapia, le silure et le machoiron sont les principales espèces qui sont élevées. L'élevage des cobayes commence également à être pratiqué.

\subsubsection{La pratique de l'élevage des cobayes}

Un individu rencontré à N'douffoukankro pratique ce type d'élevage. Il l'associe aux élevages de moutons et de bœufs. Il vient de démarrer l'exercice de cette activité et a à son actif, 18 têtes.

Photo 3: Vue d'une ferme d'élevage de cobaye à N'douffoukankro

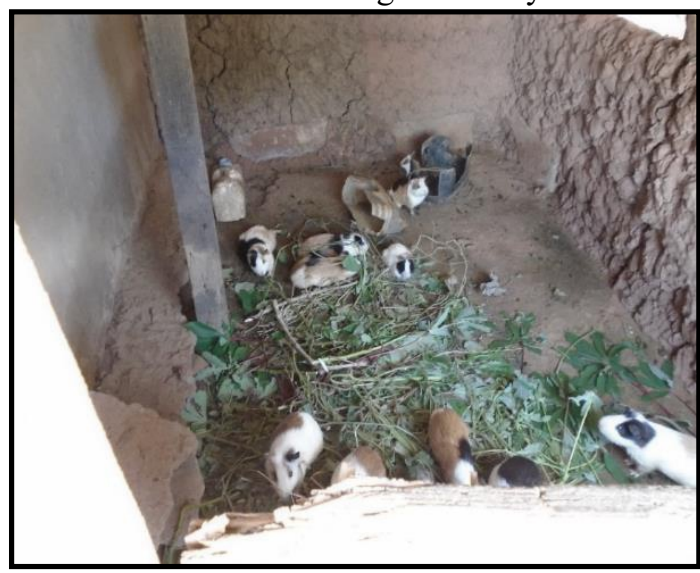

Ce type d'élevage commence à être développer autour des types conventionnels. C'est l'œuvre d'un jeune qui a commencé à l'intégrer dans ses activités d'élevage. C'est une activité qui s'inscrit dans la droite ligne de la politique de diversification des sources de revenus prônée par les jeunes. Source : Konan G., Mai, 2015 
Les exploitants qui se sont intéressés à ces types d'élevages sont au nombre de 87 personnes soit $17 \%$ (tableau 8 ) avec 72 principaux sites.

Tableau 8: Récapitulatif de la part des exploitants selon le type d'élevage pratiqué

\begin{tabular}{|c|c|c|}
\hline Type d'élevage & Nombre d'acteurs & $\mathbf{\%}$ \\
\hline Poulets & 31 & 6,15 \\
\hline Bovins & 41 & 8,13 \\
\hline Ovins et caprins & & 1,79 \\
\hline Porcins & 09 & 0,99 \\
\hline Apiculture & 05 & 0,2 \\
\hline Pisciculture & 01 & $\mathbf{1 7 , 2 6}$ \\
\hline Cobaye & $\mathbf{8 7}$ & 82,74 \\
\hline Total élevage & 417 & $\mathbf{1 0 0}$ \\
\hline Non réponse & $\mathbf{5 0 4}$ & \\
\hline Total général &
\end{tabular}

Source: Données collectées, Mai 2015

L'infériorité numérique de sites de rencontre des activités par rapport aux acteurs s'explique par le fait que, certaines personnes combinent à elles seules plusieurs activités d'élevage à la fois (tableau 9). Parmi les 72 sites de rencontre des différentes activités d'élevage, 40 fournissent déjà des revenus et 32 viennent d'abriter les activités. Chaque année de nouveaux acteurs font leur entrée dans ce secteur d'activité. Les données du tableau 9 montrent que 14 personnes parmi celles enquêtées exercent dans 02 ou 03 types d'élevage à la fois. Ce qui marque l'engouement suscité pour cette activité.

Tableau 9 : Nombre d'activité d'élevage par acteur

\begin{tabular}{|c|c|}
\hline Type d'activités & Nombre d'acteurs \\
\hline Poulet & 22 \\
\hline Poulet+pisciculture & 1 \\
\hline Poulet+cabri & 1 \\
\hline Poulet +mouton & 5 \\
\hline Boeuf & 7 \\
\hline Mouton & 13 \\
\hline Mouton+pisciculture & 2 \\
\hline Mouton+cabri & 2 \\
\hline Cabri & 0 \\
\hline Porc & 5 \\
\hline Porc+boeuf & 2 \\
\hline Porc+bœuf+cobaye & 1 \\
\hline Apiculture & 8 \\
\hline Pisciculture & 2 \\
\hline Canard & 1 \\
\hline Cobaye & 0 \\
\hline TOTAL & $\mathbf{7 2}$ \\
\hline Sorce $:$ Dore & \\
\hline
\end{tabular}

Source : Données collectées, Mai 2015 
À côté de l'élevage, il existe les activités de la pêche.

\subsection{Les activités de pêche}

C'est seul un (01) exploitant rencontré à Akoviébo qui pratique cette activité. Les activités ont lieu sur l'un des principaux cours d'eau qui longent le village (photos 4 et 5).

Photo 4: Pêche à l'aide de la technique de digue

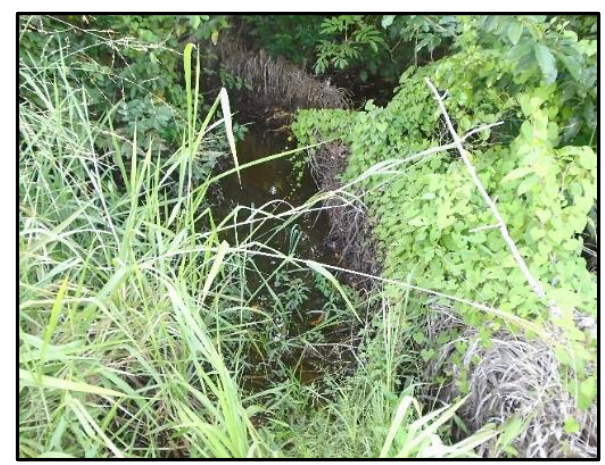

Photo 5: Pêche à la nasse

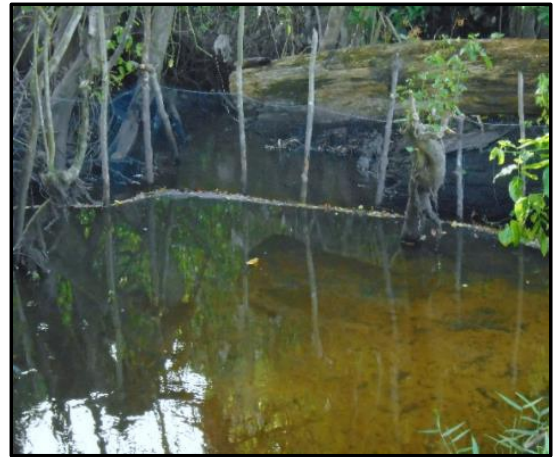

Source : Konan G., Mai, 2015

Sur la photo 4, la digue dressée en aval du plan d'eau constitue une barrière sur le trajet des poissons. Les filets sont jetés dans le périmètre circonscrit pour intercepter les poissons freinés dans leur course. La photo 5 est une nasse solidement dressée pour intercepter les poissons. Ces actions sont l'œuvre d'un pêcheur à Akoviébo. Ces techniques marchent bien car, elles permettent au piégeur d'amasser d'importantes quantités de poissons sur ces sites.

Ces activités de pêche ont lieu sur le Blo N'zué, l'une des rivières qui traversent la zone d'étude.

De petites activités commerciales sont également développées.

\subsection{Le développement du secteur informel comme complément à l'activité agricole}

L'informel désigne un ensemble d'activités non officiellement déclarées, difficilement recensées (Oura, 2010 : 282). Pour Kimbala (2008 : 30 ), ce sont l'ensemble des activités économiques légales qui échappent à toutes législations en vigueur. Il regroupe selon l'auteur, tous les secteurs économiques, primaires, secondaires et tertiaires. Et il comprend les marchands ambulants, les marchands sur étalages, les artisans tels que les réparateurs d'outils ménagers, les menuisiers, les maçons, les mécaniciens, les forgerons, les soudeurs, etc.

De par ces définitions, trois types d'activités ont été enregistrés pour ce secteur. Il s'agit des activités économiques (commerce express), artisanales et tertiaires (transport) (tableau 10). 
Tableau 10 : Types d'activités informelles exercées par les producteurs enquêtés

\begin{tabular}{|c|c|c|}
\hline Type d'activité & Effectifs & Pourcentage (\%) \\
\hline Commerce & 51 & 10,12 \\
\hline Boutique & 19 & 3,77 \\
\hline Buvette & 13 & 2,58 \\
\hline pisteur & 13 & 2,58 \\
\hline Bénévolat santé & 8 & 1,59 \\
\hline Broyage de manioc & 4 & 0,79 \\
\hline Sculpture & 4 & 0,79 \\
\hline Kiosque & 2 & 0,4 \\
\hline Transport & 4 & 0,79 \\
\hline Total & $\mathbf{1 1 8}$ & $\mathbf{2 3 , 4 1} \%$ \\
\hline Non réponse & 386 & 76,59 \\
\hline Total général & $\mathbf{5 0 4}$ & $\mathbf{1 0 0} \%$ \\
\hline
\end{tabular}

Source: Données collectées, Mai 2015

Selon les données du tableau, un effectif total de 118 producteurs soit 23,41 \% des enquêtés exercent dans ces différentes activités. Les activités commerciales (commerces express et autres) viennent en tête des activités exercées $(20 \%)$. Le transport (taxi brousse, camions kia, motos à trois roues, les pousse-pousse et les motos-taxis) et l'artisanat (la sculpture) totalisent 2 $\%$. Dans l'ensemble, le développement des activités non agricoles mobilise près de la moitié des enquêtés avec un effectif de 206 personnes soit $41 \%$.

\section{Discussion}

\section{$1 \quad$ Facteurs explicatifs de pression foncière}

Il est sans conteste que l'arrivée des déplacés entraine une pression sur l'espace (Ori B, 1988 : 37). Parlant de la question, Gaoudi P (2012 : 28), à travers une étude menée dans l'Ouest ivoirien part du fait qu'il n'existe pratiquement plus de forêt «noire » pour pratiquer de nouvelles cultures. Il poursuit pour dire que cet aspect est la manifestation de pression foncière ces dernières années. Dans la mesure où, la population agricole du côté des allochtones et des étrangers tout comme chez les autochtones croît, pendant que la ressource forestière reste statique. Il termine pour dire que cette situation exerce une pression sur la forêt. Dans la zone d'enquête, les faits politiques et les faits de société (retraite et chômage) sont apparus comme révélateur de pression sur l'espace cultivable disponible. Car, ils drainent du monde dans les villages. Dans ce sens, l'un des enquêtés rencontré à Akoviébo, affirme être retourné au village à cause de la crise militaro-politique qui a secoué le pays en 2002. Et un autre rencontré à Nangrékro quant à lui, doit son retour à la terre de la crise post-électorale de 2011. Cette proportion semble moins importante mais, elle a un impact significatif en termes d'occupation d'espaces cultivables. Car, c'est au minimum 02 hectares de 
parcelles que ces personnes occupent au moment où l'espace cultivable en luimême est soumis à une très forte pression dans les “Tos', Cette masse de retour à la terre entraine une restriction foncière. Ce qui suscite le départ de certaines personnes des villages. Le facteur démographique a également été évoqué par Coumba (2016 : 229-230), comme révélateur de pression foncière en étudiant le cas des populations déplacées au Mali par le fait du barrage de Manantali. Selon lui, la construction de ce barrage a conduit au déplacement de 33 villages et hameaux. Lors de la réinstallation, l'attribution de la taille des parcelles a été faite en fonction du nombre de personnes dans la famille. Mais, la taille du terrain n'augmente pas bien que la population ne cesse de s'agrandir (Coumba op cité : 229). Il aborde l'aspect de la distance qui sépare les villages comme élément qui favorise la pression; en prenant l'exemple de Maréna. Selon lui, ce village est installé à côté de trois villages dont Kondonia, Sobela et Nantela. Il en est également de même pour les autres villages réinstallés. Il affirme que les villages sont très proches les uns des autres (moins de $3 \mathrm{~km}$ ) dans certains cas et sont presque continus. De ce fait, à 100 $\mathrm{m}$ de part et d'autres de Maréna, il y a les terres de Kondonia et de Sobela. La pression est d'autant présente par le fait que les villages déplacés sont donc limités dans leur extension (Coumba op cité : 229). C'est le cas dans les "Tos", où la distance entre les villages déplacés n'excède guère les $03 \mathrm{Km}$. L'espace "Tos" qui a accueilli des populations déplacées enregistre aussi une croissance rapide de sa population. En effet, de 11000 habitants en 1970, elle a connu une légère baisse en 1975 avec 10958 habitants (INS, 1975). Cette baisse est liée aux mouvements de la population enregistrée dès le départ. En 1983, elle est passée à 15000 habitants ; soit un taux de croissance moyen annuel de 3,45 \% (Ori B, 1988 : 9) pour atteindre 17139 habitants en 1998 (INS, 1998). Toujours selon le RGPH 2014, on y dénombrait 29097 habitants (INS, 2014) soit une augmentation de plus du double de celle de 1970. Ce croit naturel de la population à un rythme accéléré a occasionné une pression sur l'espace disponible.

\section{Les stratégies développées par la population face à la pression foncière}

Face à la pression foncière, les populations agricoles se tournent soit vers d'autres régions où la terre est encore disponible, soit elles associent les cultures, soit elles recourent au système intensif. Tandis que les centres urbains sont la trouvaille d'une autre catégorie en l'occurrence la jeunesse, à la recherche de mieux être. Certains auteurs dont Agbroffi (2012: 5), signalait que:

Dans les années 1970, au moment de la construction du barrage de

Kossou près de Yamoussoukro, les populations Baoulé de la zone qui pratiquaient elles aussi la culture du café et du cacao furent 
déportées vers l'Ouest. Lorsque les terres du centre du pays s'appauvrirent, les paysans Baoulé

émigrèrent en masse vers l'Ouest et le Sud-ouest ; régions où les terres étaient encore fertiles.

Les paysans se dirigent donc vers les lieux où il $\mathrm{y}$ a de la forêt à défricher. Il en est de même pour Coumba (2016:230) qui a annoncé que la migration est privilégiée, parlant de l'exemple des populations réinstallées dans le cadre du barrage de Manantali au Mali. Selon lui, le barrage impose un manque de terres. Pour faire donc face à cette série de difficultés qui limitent les rendements, des jeunes, des hommes et des femmes ont choisi d'émigrer. Ils se dirigent vers Manantali ou plus loin vers les zones minières, les grandes villes du Mali ou hors du pays. Pour Ibo (2012:12), le manque de terres cultivables et l'insécurité alimentaire peuvent entraîner un exode des jeunes vers les villes en quête de meilleures conditions de vie. Brou T et al (2005 : 1), affirme que le mouvement des populations a toujours été favorisé par les potentialités offertes par le milieu d'accueil, le manque de terres cultivables dans les régions d'origine. Lassailly-Jacob (1989 : 146), affirme que face à la pression foncière à laquelle sont confrontés les déguerpis de Kossou, nombreux sont les exploitants qui ont préféré s'exiler. Ils se dirigent soit vers les régions forestières du sud du pays, soit vers leurs anciens terroirs lorsque ceux-ci sont encore exondés. Selon elle, le lac de Kossou ne s'est jamais rempli jusqu'au niveau escompté. À cet effet, il a laissé à découvert de nombreuses terres qui avaient été évacuées. Selon l'auteur, leurs anciens occupants sont retournés clandestinement les remettre en valeur. Ils justifient qu'à ces endroits, ils peuvent cultiver de vastes superficies de terres de forêt comme de savane, profiter de tous les avantages offerts par la présence d'un terroir (chasse, récolte du vin de palme, cueillette de noix de kola, de régimes de palme, de fruits, adoration des fétiches, etc.) et associer à leur agriculture des activités piscicoles. Chez les populations relogées, l'on a enregistré des départs sur les anciens sites et d'autres zones rurales ou urbaines à la recherche d'autres moyens de survie ; vu les conditions difficiles d'accès à la terre. Une étude réalisée par le Ministère de l'Agriculture (1993) dans le cadre du Plan Directeur de Développement Agricole (PPDA, 1992-2015), montre que la pression démographique et l'accroissement très important des superficies plantées en café et en cacao notamment ont amené les populations à défricher de nouvelles forêts et même à s'approprier des forêts classées. Ori (1988:19), dit que la forêt et le sol sont à la fois dégradés dans les forêts des "Tos". Ce qui est à l'origine d'un mouvement de population incontrôlé, suscité par la quête de terres pour la culture du café et du cacao. Il précise que l'exode est dirigé vers d'autres campagnes du Sud-ouest, du Centre-ouest et de l'Ouest du pays. Les paysans se dirigent alors dans d'autres régions où la forêt est encore 
disponible pour y ouvrir de nouvelles plantations (Gastellu J-M, 1978 : 40). La pression affecte également la qualité du sol et affecte les rendements agricoles. Ce qui impose l'utilisation des intrants. Dans cette optique, Coumba $(2016$ : $230 ; 232)$, affirmait que les villageois déplacés ont aussi pensé à une utilisation massive des produits chimiques. C'est en fait l'une des solutions les plus adoptées par les habitants qui avant le barrage, utilisaient moins d'engrais. Ce fait est rapporté par les villageois enquêtés qui affirmaient dans les propos :

Avant, la vie était moins chère et l'agriculture plus simple et rentable. Aujourd'hui, l'agriculture est devenue très difficile. Pour avoir de bons rendements, il faut utiliser des engrais et avoir de bons outils de travail. Le petit mil, le sorgho, l'arachide rien ne prend sans l'utilisation de l'engrais chimique.

À ce titre, Diarra S (1979 : 88), évoque que des éleveurs-défricheurs dans le canton de Gangara (Nigéria) face à l'exiguïté de l'espace disponible ont été contraint à pratiquer une agriculture intensive. Ils procèdent par l'emploi du fumier animal, par une discipline culturale rigoureuse et par une sélection judicieuse des espèces cultivées. L'auteur évoque également que la pression foncière a entrainé la reprise de la transhumance par certains éléments. Il s'agit de ceux qui ne disposent pas d'une superficie optimale pour obtenir des récoltes suffisantes en vue de faire face à leurs besoins alimentaires et monétaires (Diarra S, op cité : 89). Toujours dans la recherche d'alternatives à la pression foncière, d'autres semences plus adaptées sont vulgarisées pour impulser les productions agricoles. Car en pareille circonstance, la seule issue possible réside dans l'accroissement de la productivité des terres déjà mises en cultures. Ce, à travers l'adoption des pratiques agronomiques les plus efficaces telles que l'intensification agricole, l'introduction de variétés plus productives et résistantes aux maladies (Kouadio H et Desdoigts A, 2012 : 12). C'est ce qui explique l'importance des superficies de maïs hybride affectées aux villages “Tos" (Baikeh J, 2016 : 2). C'est le cas pour l'exploitation du cacao hybride, nouvelle semence du CNRA qui est vulgarisée par l'ANADER. Elle est devenue à cet effet, la nouvelle trouvaille des exploitants en matière d'exploitation cacaoyère. Car, c'est une semence qui résout le problème d'espaces cultivables de par son rendement élevé à l'hectare et aussi de la vélocité de sa production. Elle est actuellement, le pivot des exploitations cacaoyères dans la forêt des "Tos". De la Vaissière et Silué (1978 : 9), soutiennent que face à l'absence de nouvelles forêts, l'économie de plantation ne doit pas disparaître. Elle doit plutôt s'adapter à ce nouveau contexte en adaptant de nouvelles stratégies. Celles-ci se résument à la recherche de complémentarités entre champs de cultures pérennes et de cultures vivrières ou bien entre exploitations spécialisées dans les cultures pérennes et les 
cultures vivrières. Ces auteurs enregistrent de même une autre stratégie plus incitative. Elle consiste plutôt en un 'aménagement de l'espace' tout en compensant le manque à gagner en surface par une intensification de la surface cultivée. Aujourd'hui, la réussite de toutes exploitations agricoles chez les déplacés de "Tos" est conditionnée par une utilisation accrue d'intrants. Cette situation est le reflet de l'image de la Côte d'Ivoire, soulignée par Brou T et al (2005: 1). Ils affirmaient que le passage de la culture extensive à la culture intensive, apparait actuellement comme la seule voie pour maintenir le potentiel de production agricole du pays. L'élevage est également une alternative à la pression foncière. En ce sens, l'AIP-Bouaflé (2009), signalait que l'apiculture a été introduite dans les "Tos" par l'ONG « relève de "Tos" " sous l'action du Fonds de Développement de la Formation Professionnelle (FDFP) en 2008. Le but est de permettre aux jeunes d'être autonomes et de les rendre indépendants du café et du cacao vu la contrainte foncière dans la zone. Aujourd'hui, ils y ont associé l'élevage de volailles, bovin, porcin, ovin et caprin. L'enjeu autant suscité pour cette activité réside dans le fait qu'elle apparaît comme une alternative à la pression foncière. En même temps, elle procure des bénéfices satisfaisants aux acteurs. Généralement, les villageois érigent des abris traditionnels dans les activités d'élevage. Les bâtis de type moderne sont l'œuvre des citadins (Tanoh J-P, 2013:180). Des abris modernes ont été rencontrés uniquement à Akoviébo en élevage porcin. C'est l'œuvre d'un fonctionnaire. Il a investi dans ce secteur vu sa rentabilité. Ce sont ses petits frères restés au village qui se chargent de la gestion. Cette activité vient résorber le déficit d'espaces cultivables pour ces individus. L'association des cultures n'est pas exclue. Parlant de cette stratégie, Djossi (2009: 16), évoque la situation des petits exploitants villageois situés aux environs des agro-industries de la région du Sud-ouest du Cameroun. Il soutient que pour faire face à la pression foncière et démographique, la majorité de ces exploitants pratique un système de cultures pluri-espèces à base de plantes pérennes. L'auteur précise qu'une vingtaine de cultures vivrières sont en association avec les trois plantes pérennes (cacao, palmier à huile et hévéa). Car, d'après les exploitants, il n'existe pas d'autres parcelles consacrées aux cultures vivrières. C'est pourquoi, ils utilisent cette technique. Le but étant de maximiser l'utilisation de l'espace disponible et de diversifier la production sur la parcelle. Ceci, pour subvenir aux besoins alimentaires de la famille. Dans les villages " Tos", au sein de la proportion de ceux qui associent les cultures, $72 \%$ des exploitants expliquent la motivation pour ce système par le manque d'espaces cultivables. Tandis que $23 \%$ l'imputent à la réduction du temps de travail. Aussi, le justifient-ils par la fourniture d'ombrage pour les jeunes cacaoyers. Ce qui leur permet de se développer. 


\section{Conclusion}

Au terme de cette étude portant sur «Pression foncière en milieu rural ivoirien : quelles stratégies adoptent les paysans baoulé réinstallés dans la forêt des "Tos" à Bouaflé ? », l'on note que la pression foncière résulte de la croissance rapide de la population. Celle-ci se caractérise surtout par un fort taux de déscolarisation. Également, le retour massif de jeunes en campagne pour revendiquer le droit d'héritage va exercer une certaine pression sur le foncier. La situation s'est d'autant plus accentuée suite aux crises politiques et au chômage qui vont y déverser un nombre important de personnes en quête de meilleures conditions de vie. Cette masse de retour à la terre entraine une restriction foncière. Pour échapper à cette contrainte, le départ de certaines personnes des villages apparait comme des stratégies de contournement des contraintes liées à la pression foncière. Elles se tournent ainsi vers d'autres régions rurales à la recherche d'espaces cultivables et vers la ville en quête de conditions de vie meilleures. Par ailleurs, l'association des cultures ainsi que l'intervention des intrants sont devenues un impératif dans l'exploitation actuelle de l'espace par les producteurs. Ils s'inscrivent alors dans une agriculture intensive. Ainsi, ils se tournent vers les nouvelles variétés de cacao promues par le CNRA et le maïs hybride. D'autres paysans développent les activités non agricoles telles que l'élevage, la pêche et les activités informelles. L'élevage principalement, a été l'activité à laquelle la majorité des exploitants s'est tournée. En effet, le développement des activités non agricoles constitue une alternative à la pression foncière compte tenu de l'engouement qu'elles suscitent. Mais, beaucoup reste à faire pour ce secteur. Car, lesdites activités se développent de façon traditionnelle et sans organisation formelle.

\section{References:}

1. Agbroffi Diamoi, «Conflits ethniques en Côte d'Ivoire », Most Ethnonet Africa publications,

2. Information and communication Technologies (ICTs), Training and International Scientific Cooperation, MOST/ENA/LIMSI, 2002, 11 p.

3. Baikeh Jonas, «Sécurité alimentaire en Côte d'Ivoire : Du maïs hybride en expérimentation »soir info-l'inter, Abidjan, 2016, 2 p.

4. Brou Télesphore, Johan Oszwald, Sylvain Bigot et Éric Servat, « Risques de déforestation dans le domaine permanent de l'État en Côte d'Ivoire : quel avenir pour ces derniers massifs forestiers ?», Télédétection, Éditions scientifiques GB, numéro (1, 2,3), volume 5, Paris, 2005, pp 263-275.

5. Coumba Cissé, « vivre à l'ombre proche » du barrage de Manantali : les représentations sociales des impacts dans les campements et les villages environnants », Thèse, LADYSS, Domaine Géographie, Université Paris 8-Vincennes-Saint-Denis, 2016, 426 p. 
6. De La Vaissière Pascal, « Stratégie pionnière et stratégie d'intensification s'opposent: Le cas de la forêt des "Tos" (Bouaflé) », in Le dynamisme foncier et l'économie de plantation séminaire inter instituts, ORSTOM, CIRES, colloque, Abidjan, 1978, pp 73-82.

7. De La Vaissière Pascal et Silué Moussa, «Complémentarité ou concurrence entre cultures pérennes cultures vivrières », in Le dynamisme foncier et l'économie de plantation, colloque,

8. Séminaire inter instituts, ORSTOM, CIRES, Abidjan, 1978, pp 8-12.

9. Diarra Seydou, «Les stratégies spatiales des éleveurs-cultivateurs peul du Niger central agricole », in maitrise de l'espace agraire et développement Afrique tropicale: logique paysanne et rationalité technique, Université d'Abidjan, Côte d'Ivoire, compte rendu de débats, colloque de Ouagadougou, ORSTOM, Paris, 1979, pp 87-91.

10. Dibi Hyppolyte N'da, Kouakou Édouard, Mathieu Egnankou Wajda et Kouadio Affian, «Apport de la télédétection au suivi de la déforestation dans le Parc National de la Marahoué », Télédétection, Édition des Archives Contemporaines/Édition Scientifique GB, Gordon and Breach Scientific Publisher, numéro 1, volume 8, CURAT, Abidjan, 2008, pp 17-34.

11. Djossi Isabelle Nkapnang, «Les petits exploitants tirent profit des cultures pérennes dans le Sud-ouest Camerounais, in Agriculture durable à faibles apports externes », CARBAP, Douala/Cameroun, numéro 1, volume 25, 2009, pp 16-17.

12. Gastellu Jean-Marc, « La Course à la forêt dans le Moronou », in le dynamisme foncier et l'économie de plantation, séminaire inter instituts, ORSTOM, CIRES, Abidjan, 1978, pp33-45.

13. Gaouli Bi Anicet Patrice, «Tutorat et Conflits Fonciers Ruraux dans l'ouest Ivoirien : le Cas de Fengolo dans la Sous-préfecture de Duékoué, » CDP, Rapport de recherche du CODESRIA, numéro 16, Dakar (Sénégal), 2012, 52 p.

14. Ibo Jonas, «Phénomène d'acquisition massive des terres et dynamiques socio-foncières en milieu rural ivoirien : enjeux socioéconomiques et culturels », conférence-débat, INADES formation, Abidjan, 2012, 14 p.

15. INS (Côte d'Ivoire), « Recensement Général de la Population », 1975.

16. INS (Côte d'Ivoire), « Données sociodémographiques et économiques des localités: Résultats définitifs par localité », Région de la Marahoué, BTPR, Tome 1, Volume 3, RGPH, Abidjan, 1998, 28 p.

17. INS (Côte d'Ivoire), «Recensement Général de la Population et de l'habitation », 2014. 
18. Kimbala Akim Makiadi, «Le secteur informel comme stratégie de survie des congolais », Économie et Finance, Université KongoGraduat, 2008, 66 p.

19. Kouadio Hugues et Desdoigts Alain, « Déforestation, migrations, saturation et réformes foncières : La Côte d'Ivoire entre résilience rurale et litiges fonciers », conférence, Centre Vaugirard, Université Panthéon-Assas, Paris, 2012, 51 p.

20. Lassailly-Jacob Véronique, « Aménagements hydrauliques et droits fonciers: Le cas des “Déguerpis"de Kossou (Côte d'Ivoire), in Tropiques : lieux et liens : florilège offert à Paul Pélissier et Gilles Sautter », Collection Didactiques, ORSTOM, Paris, 1989, pp. 138149.

21. Ministère de l'Agriculture, «Plan Directeur du Développement Agricole (PPDA), 1992- 2015) », République de Côte d'Ivoire, Abidjan, 1993, $171 \mathrm{p}$.

22. Oura Raphaël, «Agriculture et urbanisation: le cas de la Souspréfecture de Bonoua », thèse

23. Unique de doctorat, Abidjan, IGT- Université FHB-Cocody (Côte d'Ivoire), 2010, $420 \mathrm{p}$.

24. Ori Boizo, «Étude sur le développement ivoirien, "Réalités et stratégies de développement"'», document de travail, SDU, numéro 15, ORSTOM, Abidjan, 1988, 179 p.

25. Ori Boizo, «Nangrékro, N'dènoukro, Attossé, Bénou, Akoviébo, in les villages AVB de l'Ayaou Sud: données sociodémographiques ", littérature grise, ORSTOM, Abidjan, 1984, 61p.

26. Tanoh Jean-Philippe, «Économie de plantation et occupation du sol dans un front pionnier de forêt : cas de la sous-préfecture de BécédiBrignan dans le sud-est de la Côte d'Ivoire », Thèse unique de doctorat, IGT, Université Félix Houphouët-Boigny, Abidjan, 2013, $372 \mathrm{p}$. 